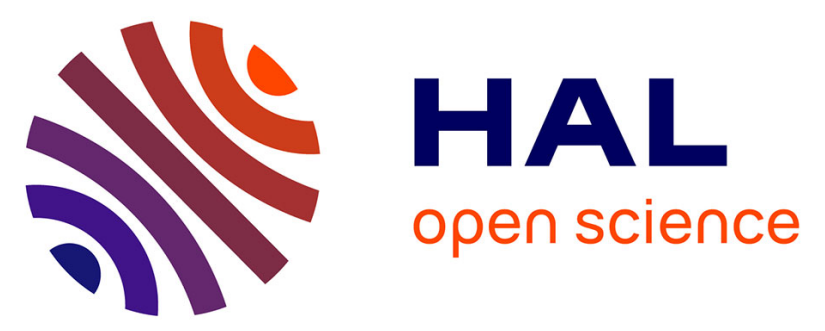

\title{
Experimental setup for fully coupled kinematic and thermal measurements at the microstructure scale of an AISI 316L steel
}

Laurence Bodelot, Laurent Sabatier, Eric Charkaluk, Philippe Dufrenoy

\section{To cite this version:}

Laurence Bodelot, Laurent Sabatier, Eric Charkaluk, Philippe Dufrenoy. Experimental setup for fully coupled kinematic and thermal measurements at the microstructure scale of an AISI 316L steel. Materials Science and Engineering: A, 2009, 501 (1-2), pp.52-60. 10.1016/j.msea.2008.09.053 . hal00367330

\section{HAL Id: hal-00367330 \\ https://hal.science/hal-00367330}

Submitted on 27 Jul 2021

HAL is a multi-disciplinary open access archive for the deposit and dissemination of scientific research documents, whether they are published or not. The documents may come from teaching and research institutions in France or abroad, or from public or private research centers.
L'archive ouverte pluridisciplinaire HAL, est destinée au dépôt et à la diffusion de documents scientifiques de niveau recherche, publiés ou non, émanant des établissements d'enseignement et de recherche français ou étrangers, des laboratoires publics ou privés.

\section{(c)(1)}

Distributed under a Creative Commons Attribution| 4.0 International License 


\title{
Experimental setup for fully coupled kinematic and thermal measurements at the microstructure scale of an AISI 316L steel
}

\author{
Laurence Bodelot, Laurent Sabatier, Eric Charkaluk*, Philippe Dufrénoy \\ Laboratoire de Mécanique de Lille, UMR CNRS 8107, Avenue Paul Langevin, 59655 Villeneuve d'Ascq cedex, France
}

\begin{abstract}
In polycrystalline metallic materials, above a certain threshold, mechanical deformations induce plastic strains at the grain scale. The development of plasticity also triggers a thermal dissipation due to local mechanical irreversibilities. These phenomena conduct to a heterogeneous state of deformation and temperature which has never been simultaneously observed at the micrometric scale. In this paper, an original experimental setup is presented in order to obtain fully coupled measurements. The objective is to get at the same time strain and temperature fields in the same zone at the microstructure scale of an AISI 316L austenitic stainless steel specimen during a tensile test. The fully coupled measurements underscore the relationship between the local plastic and thermal heterogeneities during the first stages of the deformation until a more general plastic state of the specimen. These results may also be used to perform energy balances at the micrometric scale.
\end{abstract}

\section{Introduction}

Many isotropic metallic materials are made of an aggregate of grains having a distribution of crystallographic orientations without any outstanding texture effect. During the first stages of an applied mechanical loading, according to their orientation with respect to the loading axis, some grains are submitted to a more important deformation than others, leading to local heterogeneous plasticity. By increasing the loading, the plastic yielding tends to spread over the sample and a more homogeneous state is generally observed as far as the number of grains submitted to plasticity is concerned [1,2].

Since the pioneering work of Farren and Taylor [3,4], it is also well known that this plastic deformation is accompanied by a heat dissipation conducting to temperature variations during the loading of the specimen. These historical experiments, carried out on mild steels, aluminium and copper, consisted in monotonic tensile and torsion tests during which either thermocouples or a calorimeter was used to evaluate the mean value of the heat generated during cold working and thus to realize energy balances at the scale of the specimen.

Similar quasi-static experiments have been renewed more recently using the same type of calorimetric approach $[5,6]$. However, as calorimeters remain awkward to handle, thermal mea-

\footnotetext{
* Corresponding author. Tel.: +33 3206760 86; fax: +33 320335393.

E-mail address: eric.charkaluk@univ-lille1.fr (E. Charkaluk).
}

surements have later been obtained by infrared thermography (IRT) [7]. Moreover the higher recording frequency provided by this technique enables the establishment of energy balances for specimens submitted to dynamic phenomena [8,9]. In most of these studies, thermal fields are averaged over the whole sample in order to evaluate mean thermal sources.

However, dissipation and strain fields in a polycristal are not homogeneous. A way to account for this is to perform full-field measurements. Hence, such experimental studies have been developed since a few years. For instance, thermal fields grabbed by IRT were studied to investigate local instabilities like Portevin-Le Chatelier effect [10] and kinematic fields were obtained through digital image correlation (DIC) to analyse localization phenomena like necking or Lüders bands [11]. In order to underscore the link between thermal and kinematic behaviours, couplings of IRT and DIC measurements were attempted either on both sides of the sample [12,13] or on separate samples [14]. These results were exploited to perform energy balances at the scale of specimens submitted to instable and local phenomena.

Nevertheless, some particular cases require the realization of such energy balances at the grain scale. For example, in the case of incoming plasticity or fatigue, plastic strains can be very small and heterogeneous. Performing thermomechanical measurements on a metallic sample at this scale could indeed be useful, as it would give information on the behaviour of a representative elementary volume (REV) of a polycristal.

The objective of this study is thus to focus on the REV scale and to measure, on the same surface area and at the same time, thermal 
emissions and strain fields. For that purpose, an original fully coupled thermal and kinematic measurement technique is proposed. To our knowledge, such a technique has never been developed nor presented before.

The outline of this article is therefore the following. The full-field measurements methods are first presented, namely infrared thermography (IRT) for temperature measurements and digital image correlation for strain field measurements. Next, since performing fully coupled measurements at a micrometric scale is a very delicate task, the difficulties to overcome and the precautions to take are developed. Finally, thermal and kinematic coupled information obtained during a uniaxial tensile test on AISI 316L austenitic stainless steel are presented and discussed.

\section{Experimental setup}

The aim of this section is to present, on the one hand the original setup developed for the fully coupled measurements and, on the other hand, the difficulties to overcome as well as the necessary precautions to take.

\subsection{Infrared thermography and temperature field measurements}

Any point of an object at a temperature greater than $0 \mathrm{~K}$ emits an infrared radiation invisible to the naked eye, which can be related to its temperature; infrared cameras are based on this principle. Historically, infrared detectors were developed for military purposes during the second half of last century. In the early 60s, cameras dedicated to civil applications only disposed of a single infrared detector and a complete scan of the surface was necessary to get a temperature field. As a consequence, recording frequencies were very low and measurements could suffer from thermal drift. In the 80 s, focal plane array (FPA) cameras became widespread; made of an array of detectors, they allow higher recording frequencies. The thermal radiation received by the infrared detectors generates an electric signal which is converted into an image corresponding to a map of thermal emissions and whose unit is the "digital level".

The camera used in this work is a Cedip Jade; its infrared sensitive part is a FPA of $320 \times 240 \mathrm{InSb}$ detectors, cooled down at $77 \mathrm{~K}$ by a rotary Stirling and working in the spectral range from 3 to $5 \mu \mathrm{m}$. Its maximum recording frequency in full frame is $170 \mathrm{~Hz}$ and the dynamic range of digital levels is 14 bits. This camera is used with a high magnification lens known as G1 since the spatial resolution matches the size of one detector, namely $30 \mu \mathrm{m} \times 30 \mu \mathrm{m}$; this leads to a $9.6 \mathrm{~mm} \times 7.2 \mathrm{~mm}$ observation zone. Nevertheless, such a lens induces optical distortions especially on the borders of the image, narrowing the working area to a central square of $5 \mathrm{~mm} \times 5 \mathrm{~mm}$.

The conversion of digital levels into temperatures needs two crucial steps: the calibration of the detector array and the determination of the emissivity of the sample's surface. Moreover, in order to perform accurate quantitative infrared thermography, in addition to these two steps, some precautions must be taken.

On the one hand, the calibration law, which gives a link between temperatures and thermal emissions, has to be established by exposing the detector array of the camera in front of an extended blackbody at different controlled temperatures. However each detector possesses its own behaviour which is even different each time the camera is switched on; this is a particular drawback of FPA cameras. Thus, in a standard use, the response of each detector is corrected by a gain and an offset in order to be closer to the array's mean response: this is the Non Uniformity Correction (NUC) procedure. Despite such a correction, if a detector's response is still

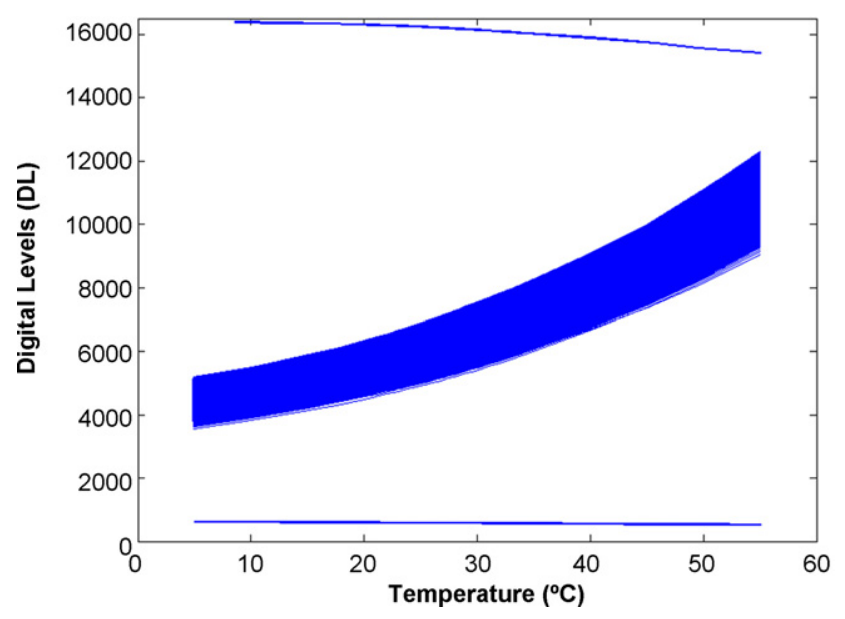

Fig. 1. Cluster of curves obtained during the calibration of the 76,800 detectors of the array. The outstanding curves at top and bottom correspond to defective detectors.

too far from the mean value, its response is replaced by one of the closest detector among those following the mean response or by an average made over several ones: this is the Bad Pixel Replacement (BPR) procedure. In many general applications, such corrections (NUC and BPR) are systematically applied. The NUC and BPR procedures tend to make the response of the detectors array uniform, which is an important disadvantage if one expects to observe small heterogeneities at a local scale or to calculate gradients or Laplacians present in the heat equation over several thermal images [15]. Nonetheless the standard NUC and BPR procedures can be bypassed by the determination of a calibration law made for each detector [16]. In this configuration, the unique mean calibration law is replaced by a set of 76,800 calibration laws. In Fig. 1, the calibration curves obtained for all the detectors are plotted in order to emphasize the heterogeneity of their response. One can notice a similar trend for all the curves except for two outstanding ones (at the top and at the bottom of Fig. 1) corresponding to defective pixels. Such pixels will not be taken into account during the data processing. Moreover, as the calibration is influenced by many parameters (distance to the object, lens used, working temperature, integration time, frame rate ...), a new calibration is necessary for each test configuration [17] but also each time the camera is switched on.

On the other hand, the emissivity of the object has to be determined. This can be realized through a classically used method consisting in a comparison between the radiation of the object and the one of an extended blackbody, both being at the same temperature [18].

At last, for a better accuracy, the camera is turned on more than $4 \mathrm{~h}$ before beginning any measurements including calibration in order to get rid of thermal drift. The working place around the camera is protected from any reflections of the environment thanks to black tissue. Every element close to the camera is also covered by black tissue or painted with high emissivity black paint in order to dispose of any residual reflection.

\subsection{Digital image correlation and strain field measurements}

Images of the sample are grabbed during the tests thanks to a CCD camera Jai CV-M4+. This camera is made of a matrix of $1368 \times 1024$ detectors, whose size is $6.45 \mu \mathrm{m} \times 6.45 \mu \mathrm{m}$. It is sensitive to visible wavelengths from 0.38 to $1 \mu \mathrm{m}$ and delivers 10 bits greyscale images. A Tamron 23FM50SP $50 \mathrm{~mm}$ lens associated to extension tubes allows a high magnification and a spatial reso- 
lution of $6.5 \mu \mathrm{m} \times 6.5 \mu \mathrm{m}$ per pixel, leading to a working zone of approximately $8.9 \mathrm{~mm} \times 6.7 \mathrm{~mm}$. For this configuration, no spatial distortion near to the borders is observed; nevertheless, the working zone is reduced to $5 \mathrm{~mm} \times 5 \mathrm{~mm}$, in order to fit the infrared data (Section 2.1).

Displacement and strain fields are obtained through DIC performed on the images captured during the mechanical deformation of the sample. The displacement field of one image is determined with respect to a reference image thanks to the DIC software Correli $^{\mathrm{LMT}}$ developed at the "Laboratoire de Mécanique et Technologie" (LMT) of Cachan in France. The principle of DIC is the following. The reference two-dimensional image is divided into square regions called zone of interest (ZOI). Then, the software matches these ZOI between one image at a time $t_{\mathrm{i}}$ and the reference image at a time $t_{0}$ thanks to a pattern recognition based on a Fast Fourier Transformation algorithm [19]. Finally, the results of the DIC analysis are two-dimensional displacement fields for each time $t_{\mathrm{i}}$, from which derive the strain fields.

The correlation of ZOIs requires the existence of a random pattern which has to cover a wide range over the greyscale. In some particular cases, such a random pattern appears naturally on the tested material so that correlation can be performed directly on images of the raw material. For example, DIC was recently computed over images of the metallic phases of polished and electrochemically etched duplex stainless steel [20]. In more general cases, the pattern has to be created artificially: the sample is usually covered with black and white paint in order to obtain a random speckle. In the present case, a coating is applied considering the sample is polished as a mirror.

\subsection{Fully coupled measurements}

As already mentioned, in order to get strain and temperature fields at the same time, a sample can be observed on one side with a CCD camera, in order to perform DIC, and on the other side with an IRT camera, in order to determine its temperature. To process these data, it must be assumed that the thermal and kinematic responses are the same on both faces of the sample [12]. In this particular case, where relatively large strains are obtained and where mean values over the whole gage zone i.e. macroscopic data are concerned, this hypothesis seems to be realistic. However, since the objective of the present work is to get the same information at the grain scale, the former method is not suitable since the microstructure is different from one face to the other. As a result, it is necessary to observe the same area of the sample simultaneously with both IRT and CCD cameras: this is the objective of the original coupling setup developed in this study.

\subsubsection{Principle of the coupling}

In order to realize this simultaneous observation, a "filtermirror" (dichroïc mirror) is placed in front of the sample making an angle of $45^{\circ}$ with respect to the sample's surface (Fig. 2a). On the one hand, this "filter-mirror" behaves like a filter since it lets only the infrared radiation go towards the IRT camera placed ahead of the sample. On the other hand, it also behaves like a mirror since it reflects the rest of the radiation, among which the visible radiation, in the direction of the CCD camera (Fig. 2).

Thanks to this particular technique, the observation of the same zone simultaneously seems to be quite easy. Nevertheless the transmission of the infrared radiation is only partial through the filter; its transmission curve depends on the wavelength and has to be taken into account during the calibration. This implies that during calibration, the "filter-mirror" has to be placed in front of the blackbody, exactly in the same configuration than for the tests.
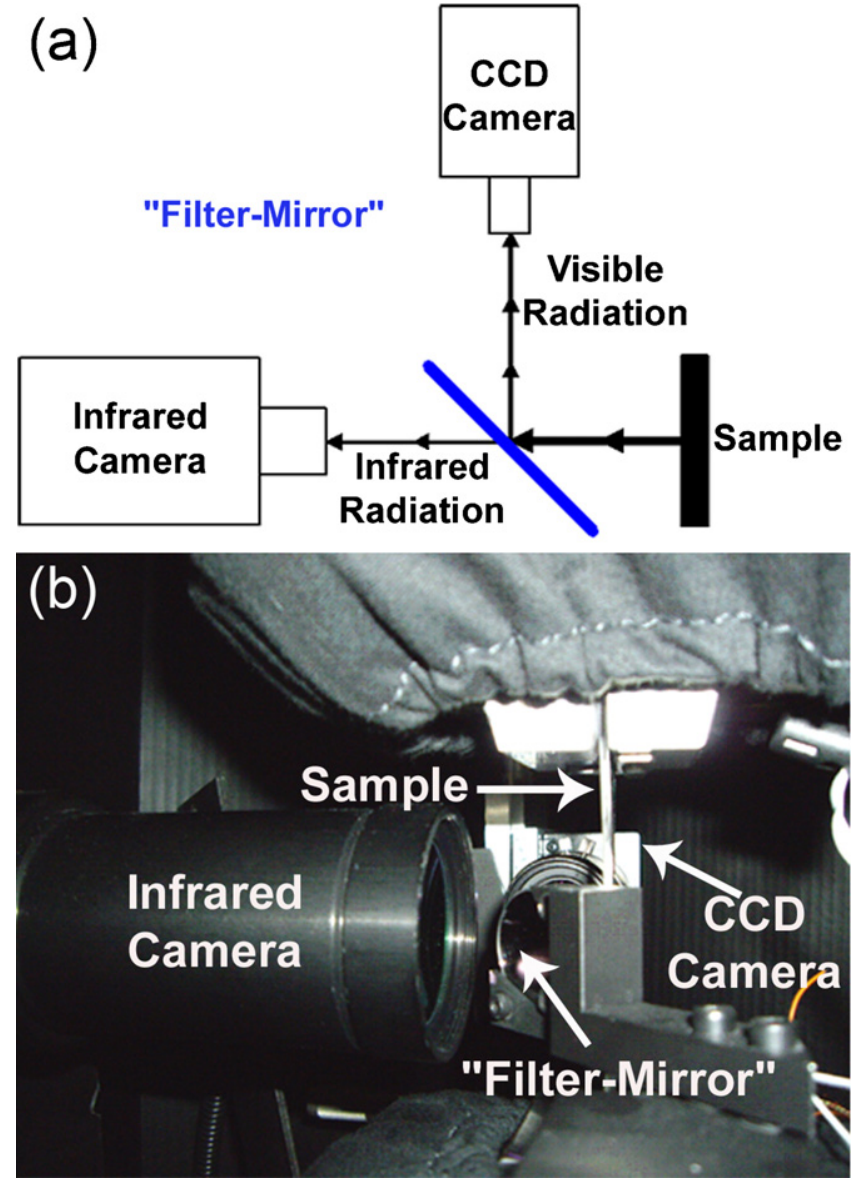

Fig. 2. (a) Principle of the coupling thanks to the "filter-mirror" and (b) view of the experimental device.

\subsubsection{Need for a special coating}

The use of this "filter-mirror" also entails overcoming another difficulty. As it was previously underlined, DIC has to be performed on optical images of random and contrasted grey aspect, usually obtained by applying a speckle on the sample. On the opposite IRT measurements require a high emissivity thus dark, and uniform surface classically obtained by applying mat black spray paint on the sample. These coatings are inconsistent from a macroscopic point of view, when observed by both cameras equipped with standard lenses (Fig. 3). If the black paint coating is observed with each camera with the high magnification lenses described previously, the result is even worse. Black paint seen by the CCD camera at this scale seems to provide a speckle (Fig. 4a) but the spots are too large to perform DIC at a microscopic scale. Moreover the histogram of grey levels (1024 levels) of this image gives two peaks localized on the corresponding black and white part of the scale (Fig. 6a); accordingly this image is not suitable for DIC. The black paint coating observed by the IRT camera equipped with a G1 lens (Fig. 4b) has not the same properties as if observed by a standard lens (Fig. 3b). At this scale the image is neither dark nor homogeneous. The detectors are mirrored in the coating; this effect, commonly named "Narcissus effect", is characterized by the round dark patch in the middle of the image (Fig. 4b).

As a consequence, the development of a special coating is necessary to get optical and infrared images simultaneously at the micrometric scale. It has to be suitable, at the working scale i.e. with the magnifications used, for both kinematic and thermal field measurements. This original coating seen by the CCD camera (Fig. 5a) 

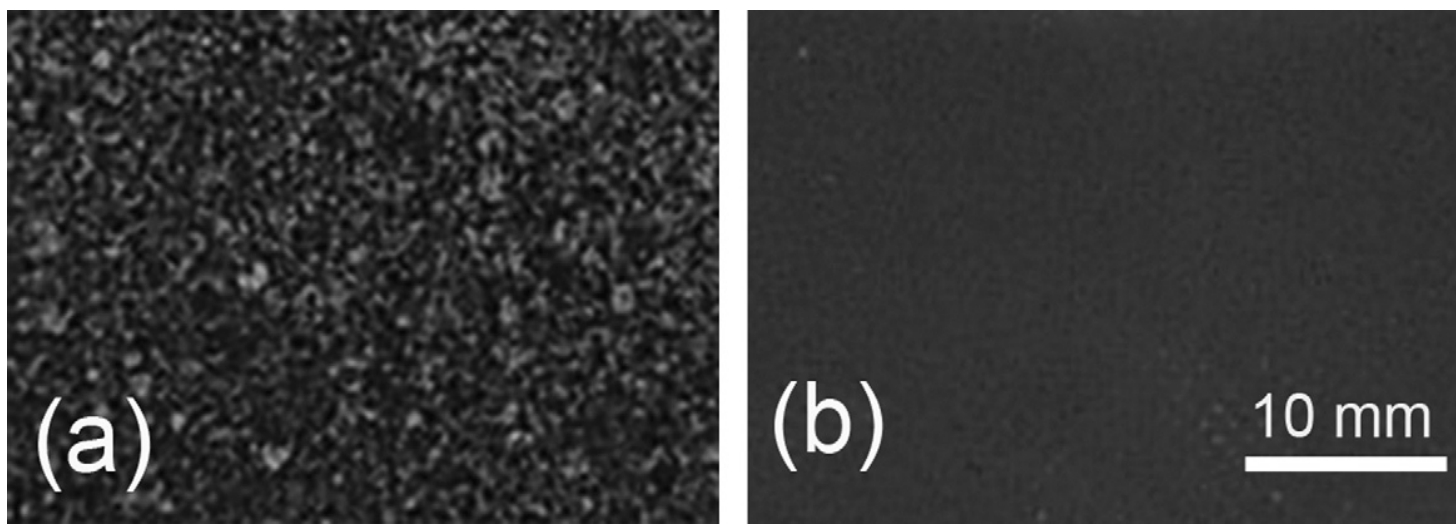

Fig. 3. (a) Speckle of paint observed by the CCD camera and (b) black paint coating observed by the IRT camera. Both cameras are equipped with standard lenses.
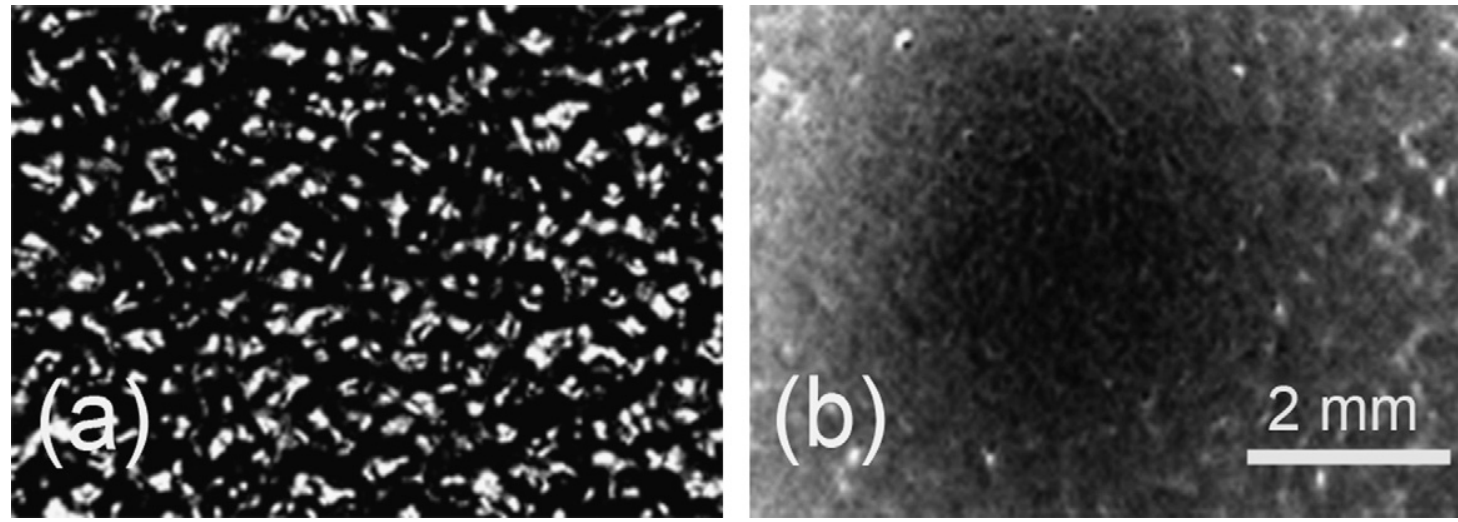

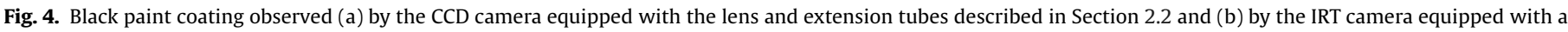
G1 lens. The round dark patch in the middle of this image is due to the reflection of the detector array in the coating ("Narcissus effect").

has a better aspect as a speckle than paint (Fig. 4a) and the histogram of grey levels covers a large range over the 1024 ones (Fig. 6b). When observed by the infrared camera equipped with a G1 lens, it exhibits a dark and homogeneous aspect having a scatter of about 700 DL out of 16,384 over the image (Fig. 5b) and the "Narcissus effect" which appeared in the case of black paint (Fig. 4b) is annihilated.

To sum up this section, the simultaneous observation of a given zone by IRT and CCD cameras is made possible thanks to the "filter-mirror" transmitting the infrared radiations and reflecting the visible ones. High magnification lenses give access to a high spatial resolution and then to information at a micrometric scale.
But the keystone of the technique is the specially developed coating since it enables the simultaneous fully coupled measurement of two data that require, at first sight, different kinds of coating.

\section{Experiments}

\subsection{Specimen material}

The choice of the material is also a crucial issue. Firstly, the material's thermal diffusivity (ratio of thermal conductivity to volumetric heat capacity) has to be as low as possible so that heat generation is not dissipated nor homogenised too quickly with
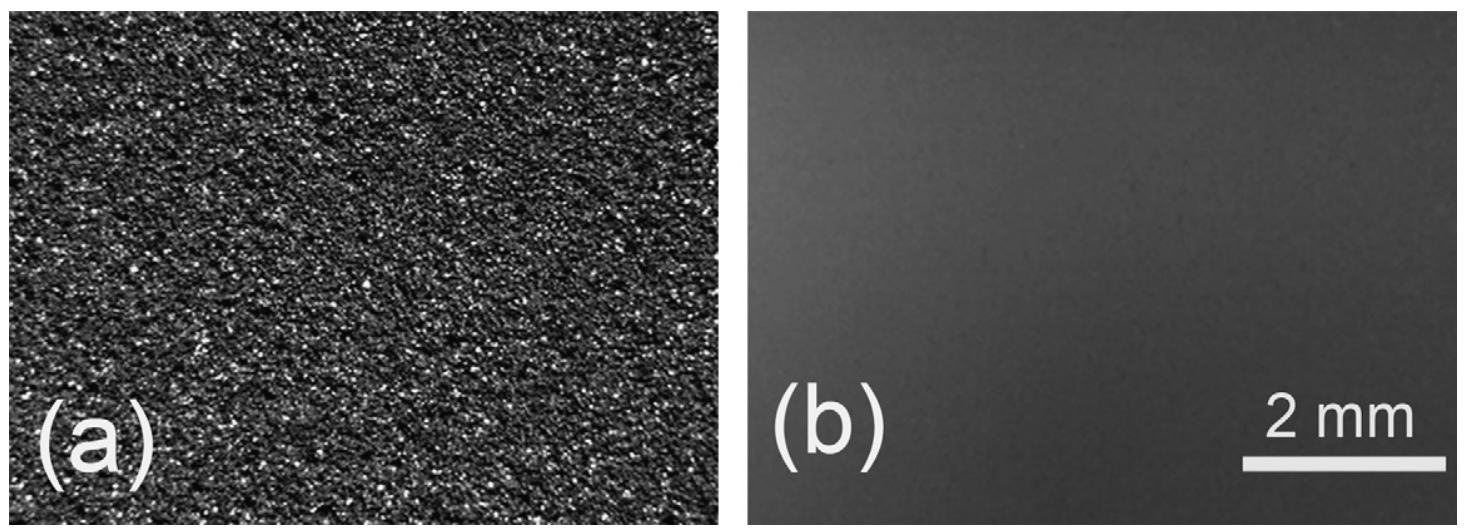

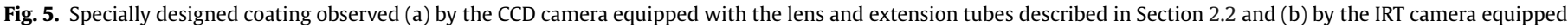
with a G1 lens. 

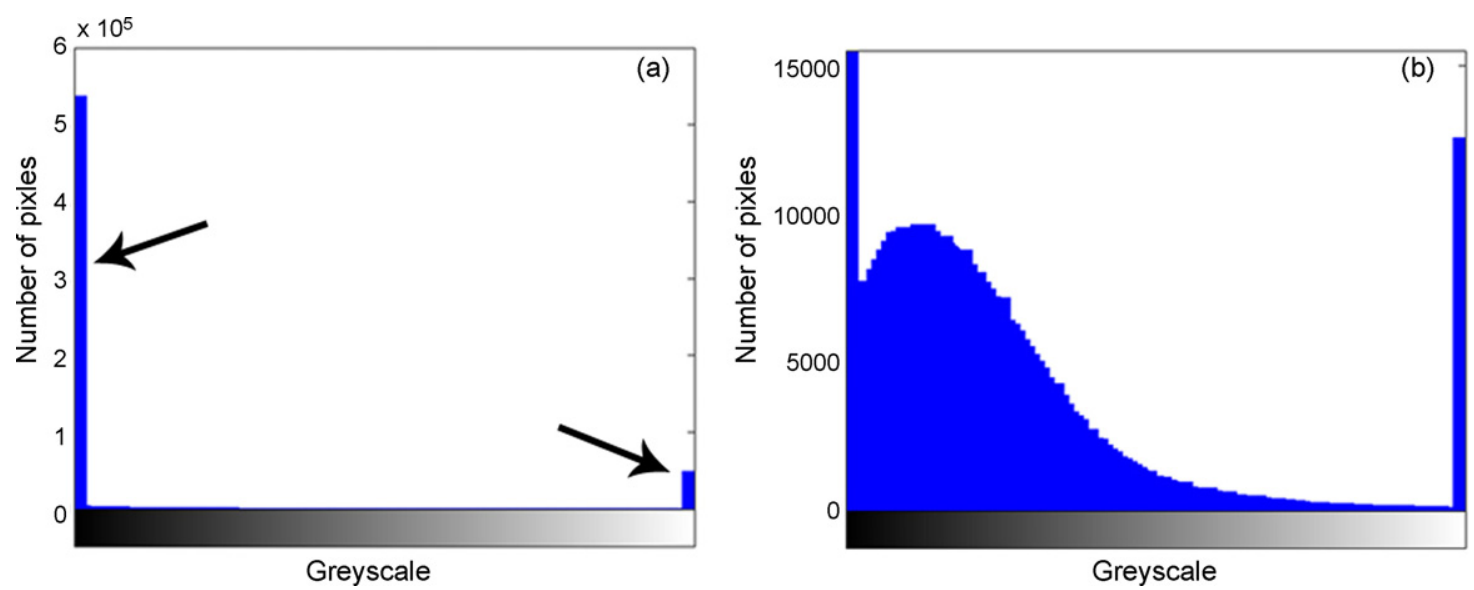

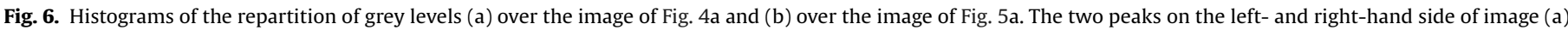
correspond to the black and white parts of the scale.

Table 1

Thermal properties for several metals at $20^{\circ} \mathrm{C}[21,22]$.

\begin{tabular}{lllll}
\hline Metal & $\begin{array}{l}\text { Density } \\
\left(\rho, \mathrm{kg} \mathrm{m}^{-3}\right)\end{array}$ & $\begin{array}{l}\text { Specific heat } \\
\left(c_{p}, \mathrm{~J} \mathrm{~kg}^{-1}{ }^{\circ} \mathrm{C}^{-1}\right)\end{array}$ & $\begin{array}{l}\text { Conductivity } \\
\left(k, \mathrm{~W} \mathrm{~m}{ }^{-1}{ }^{\circ} \mathrm{C}^{-1}\right)\end{array}$ & $\begin{array}{l}\text { Diffusivity } \\
\left(\alpha, \times 10^{5} \mathrm{~m}^{2}{ }^{\circ} \mathrm{C}^{-1}\right)\end{array}$ \\
\hline Silver & 10,500 & 230 & 418 & 17.1 \\
Copper & 8,940 & 380 & 389 & 11.4 \\
Aluminium & 2,700 & 860 & 200 & 8.6 \\
Brass & 8,500 & 370 & 100 & 3.3 \\
Steel (0.1\%C) & 7,850 & 490 & 46 & 1.2 \\
AISI 316L steel & 8,000 & 500 & 15 & 0.4 \\
\hline
\end{tabular}

respect to the frame rate of the IRT camera. This is actually essential to be able to capture the thermal field's heterogeneities. Secondly, single phase steel is preferred, such as the thermal properties are homogeneous in all grains. Finally, the grain size has to be large enough so that several pixels of both IRT and CCD detectors arrays can stare at one grain. This last condition leads to an average grain size of more than $100 \mu \mathrm{m}$.

In order to respect as well as possible all the above-mentioned constraints, the chosen material is an AISI 316L austenitic stainless steel since it meets the two first criteria: its diffusivity is low (Table 1) and it is a single phase steel. Its initial grain size is about $10 \mu \mathrm{m}$, which is not enough compared to the constraint of roughly $100 \mu \mathrm{m}$. However, in the case of AISI 316L, the average grain size can be easily raised up to $130 \mu \mathrm{m}$ (data determined by the linear intercept method considering twins) thanks to a heat treatment at

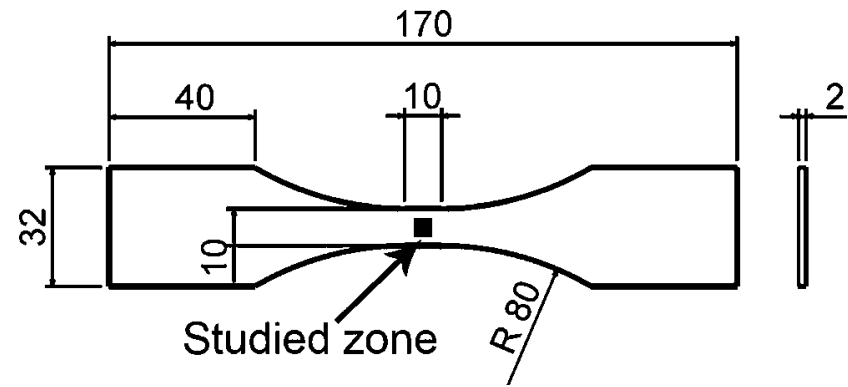

Fig. 8. Geometry of the tested plate samples. The black square in the middle of the gage zone corresponds to the $5 \mathrm{~mm} \times 5 \mathrm{~mm}$ studied zone.

$1200^{\circ} \mathrm{C}$ under air for $2 \mathrm{~h}$, immediately followed by a water quench Samples of the material before and after the heat treatment are presented in Fig. 7 to underscore the grain growth. Samples were chemically etched before being examined under a microscope.

As a result, samples are obtained as follows: $3 \mathrm{~mm}$ thick specimens are cut out by electro-erosion from a thick laminated sheet of 316L. They are heat treated under air as described above and the oxide layer created all around the sample is removed during the step of polishing. Actually the samples are mechanically polished up to $1 \mu \mathrm{m}$ on both faces, and up to grit $4000 \mathrm{SiC}$ paper on the edges. Final samples are thin plates of $2 \mathrm{~mm}$ of thickness, having a gage area of $10 \mathrm{~mm} \times 10 \mathrm{~mm}$ (Fig. 8). The last operation consists in
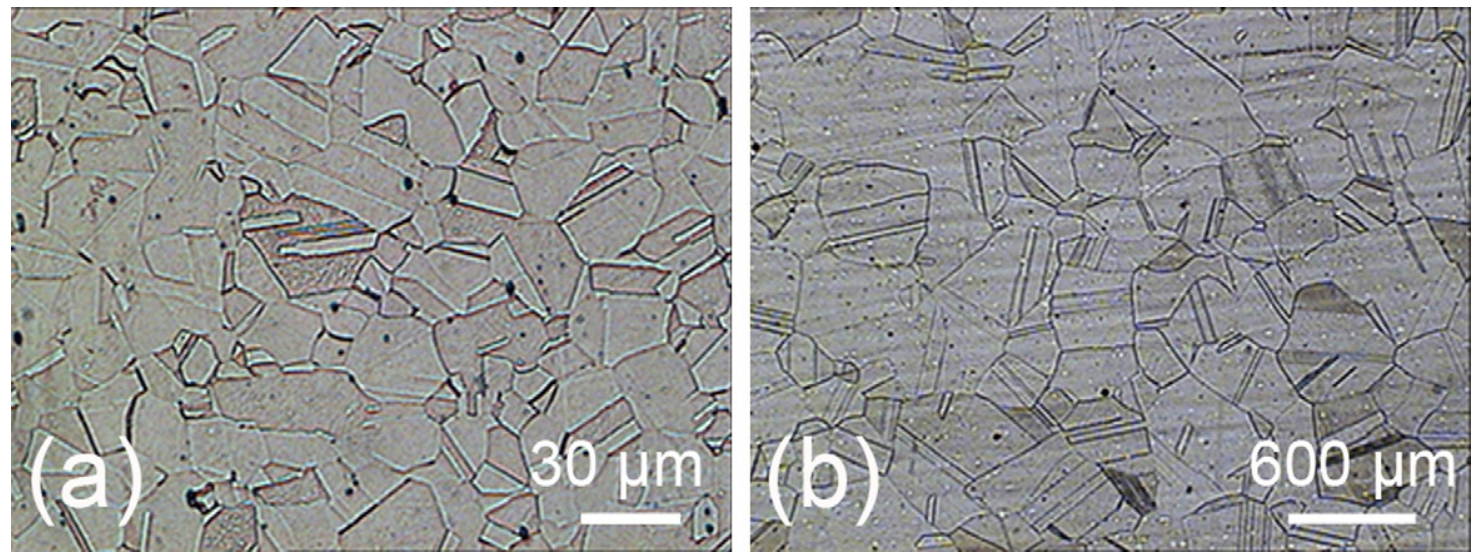

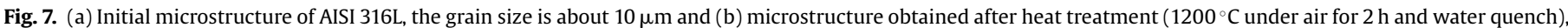
the grain size is now about $130 \mu \mathrm{m}$. 


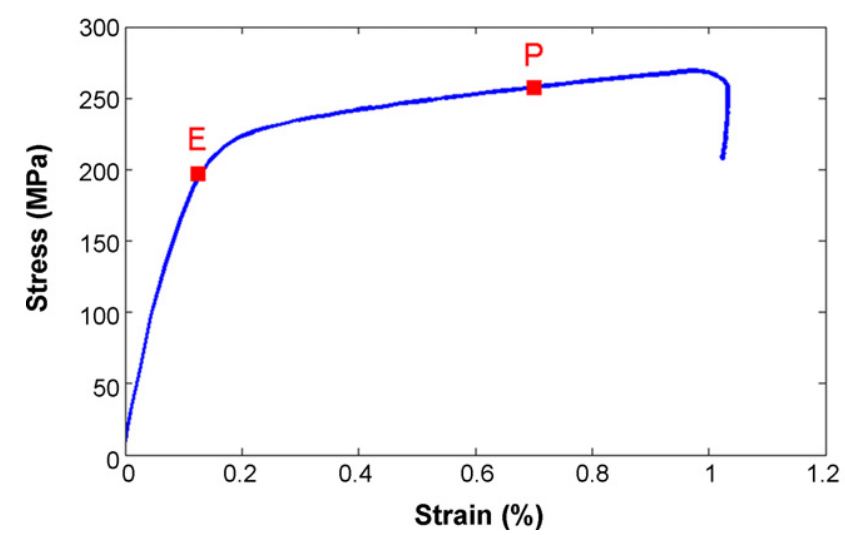

Fig. 9. Stress-strain curve and pointing out of two instants being studied further: E corresponds to a global elastic state of the sample and P corresponds to a global plastic state.

the application of the specially designed coating over the sample (Section 2.3).

\subsection{Testing}

Mechanical tests are performed on an electro-mechanical Instron 4508 machine which allows tests up to $300 \mathrm{kN}$ on a speed range from 0.1 to $100 \mathrm{~mm} / \mathrm{min}$. The test carried out in this work is a uniaxial tensile test at $23.4 \mathrm{~mm} / \mathrm{min}$. The objective is the validation of the experimental setup in order to answer the following question: is it possible to distinguish heterogeneities in the kinematic and thermal fields or not?

During the test, displacement, strain and temperature fields are recorded within the gage zone thanks to the fully coupled measurement technique presented in the previous section. The load applied to the sample, as well as the displacement of the crosshead, is also recorded during the tests.

In order to disturb as less as possible the thermal measurements, the working zone is protected from its environment as it has already been explained, light is turned off and the room temperature is kept steady thanks to air conditioned.

\subsection{Results}

The stress-strain curve obtained during the test is plotted in Fig. 9. On this curve, two instants are arbitrarily chosen and will be
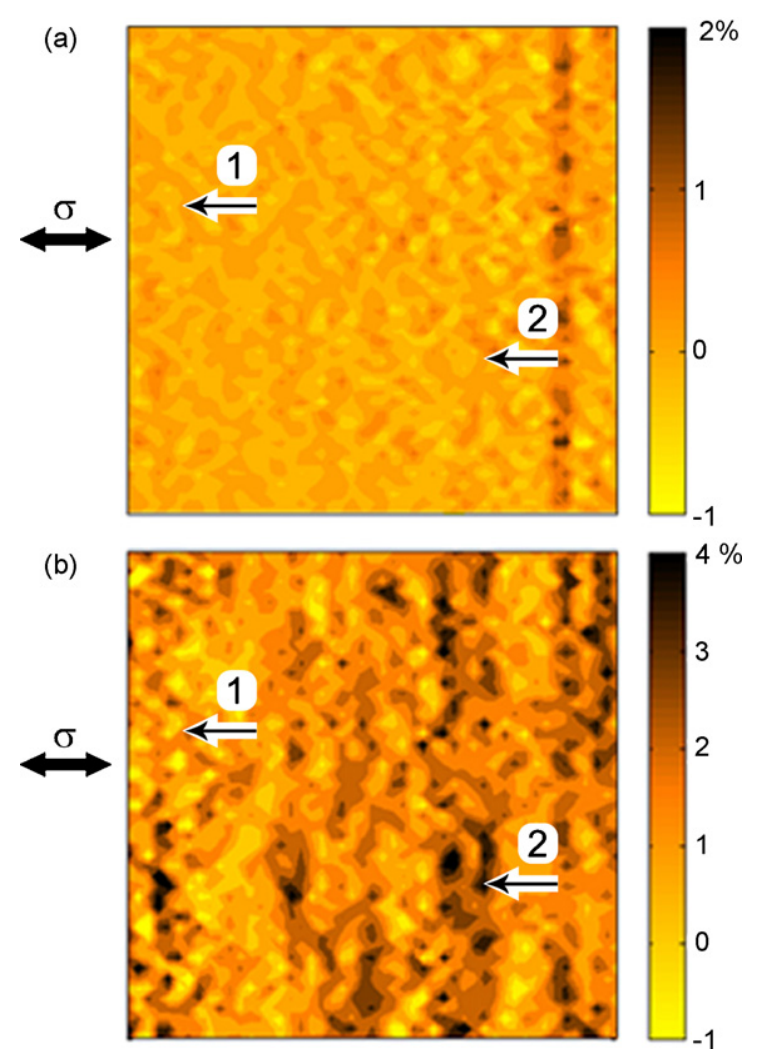

Fig. 10. Axial strain fields obtained by DIC (a) at level E (Fig. 9) within the elastic domain and (b) at level P (Fig. 9) whereas plasticity has already occurred. Points 1 and 2 are submitted to very different levels of strain.

studied: the first, marked E, corresponds to a global elastic state of the sample; the second, marked $\mathrm{P}$, corresponds to a global plastic state.

The axial strain field of the elastic state marked E in Fig. 9, as well as one of the plastic state marked P, is presented in Fig. 10. They are obtained by DIC; the size of the ZOI is $16 \times 16$ pixels, leading to a spatial resolution of $104 \mu \mathrm{m} \times 104 \mu \mathrm{m}$. The studied zone is a $5 \mathrm{~mm} \times 5 \mathrm{~mm}$ area in the middle of the gage zone (Fig. 8).

The distributions of strain values of the previous fields are plotted in Fig. 11. On the histogram of Fig. 11a, the mean strain is $\varepsilon=0.10 \%$ and the standard deviation is equal to $0.29 \%$. On the his-
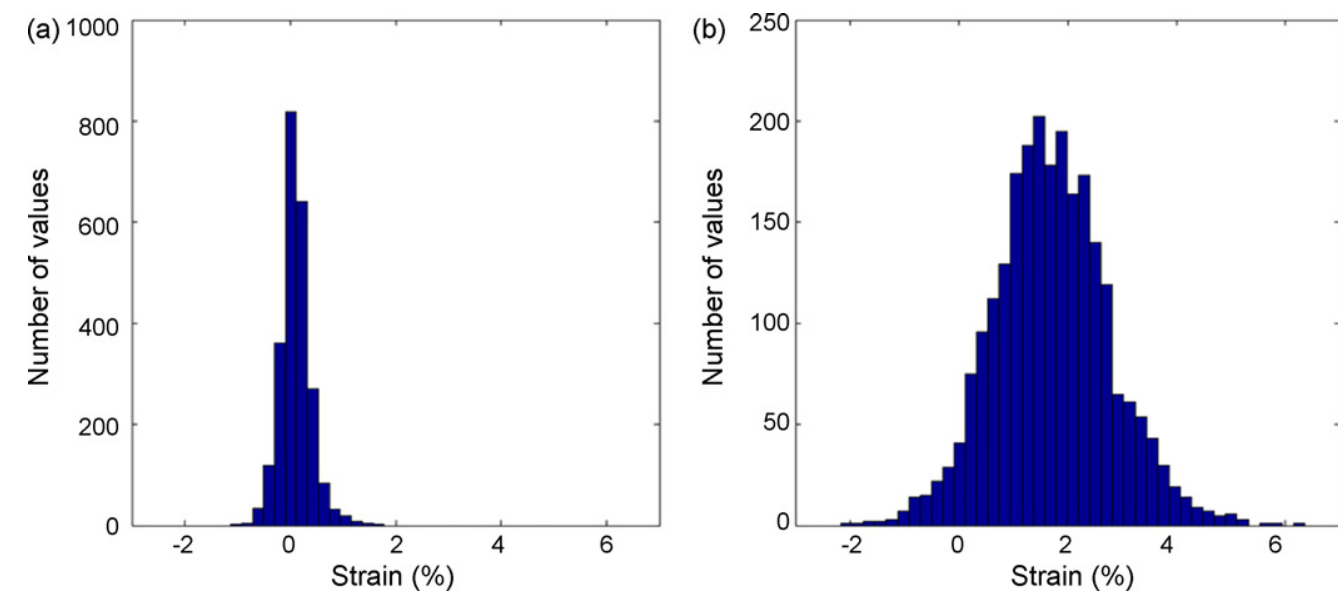

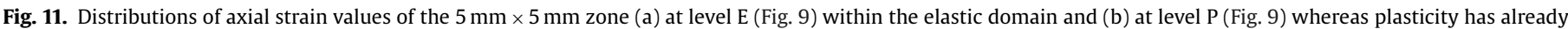
occurred. 

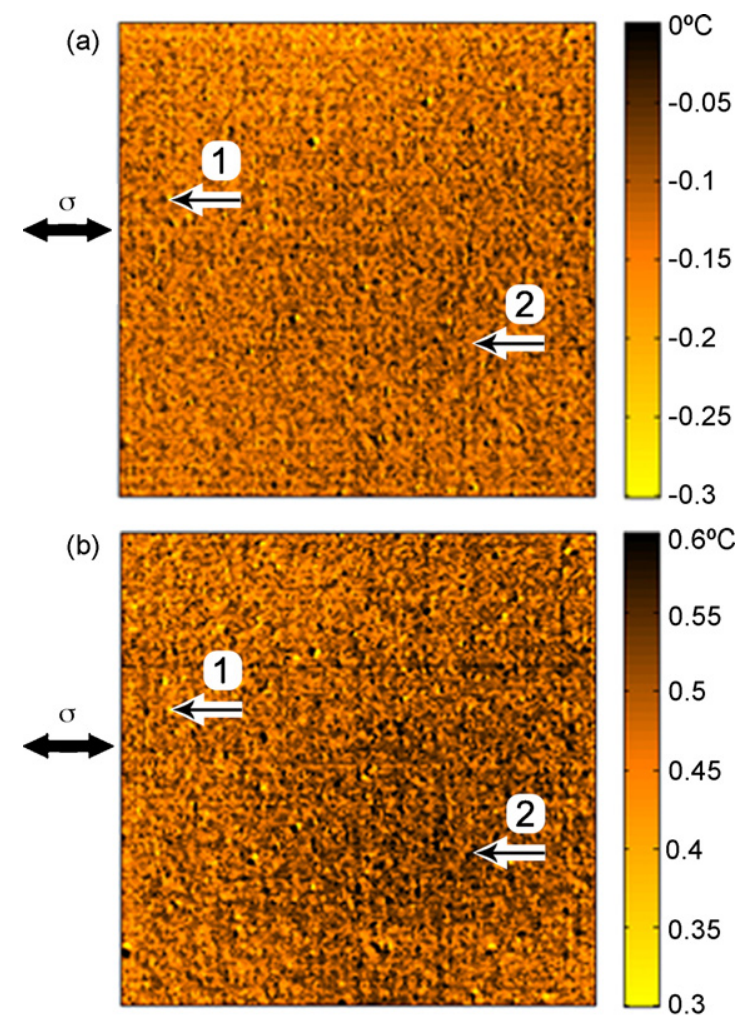

Fig. 12. Temperature fields obtained by IRT (a) at level E (Fig. 9) within the elastic domain and (b) at level P (Fig. 9) whereas plasticity has already occurred. Points 1 and 2 of Fig. 10 are transferred to the thermal fields.

togram of Fig. $11 \mathrm{~b}$, the mean strain is $\varepsilon=1.71 \%$ and the standard deviation is equal to $1.07 \%$.

Temperature fields captured at the same instants $\mathrm{E}$ and $\mathrm{P}$ than the kinematic fields are presented in Fig. 12. They correspond to the evolution of the temperature variations $\Delta T(x, y, t)=T(x, y, t)-T_{0}(x$, $y$ ), where $T_{0}(x, y)$ is the equilibrium state reached before the loading of the specimen. These variations are computed in the middle of the gage zone, over the same $5 \mathrm{~mm} \times 5 \mathrm{~mm}$ zone than the strain fields (Fig. 8). At instant $\mathrm{E}$ of the elastic domain, the temperature has dropped since the mean temperature variation of Fig. 12a is $\Delta T=-0.11^{\circ} \mathrm{C}$. At instant $\mathrm{P}$ when plasticity has occurred, the temperature has raised since the mean temperature variation of Fig. 12b is $\Delta T=0.51{ }^{\circ} \mathrm{C}$. Both fields obtained under a global elastic state of the sample and then under a global plastic state, are heterogeneous. Nevertheless, it is awkward to establish a direct link between the strain fields of Fig. 10 and the thermal fields of Fig. 12.

As a consequence, the two points marked 1 and 2, are chosen in Fig. 10, in order to determine their thermal evolution versus time. These points are submitted to very different levels of strains. The thermal evolution of points 1 and 2 spotted out in Fig. 10b are plotted in Fig. 13. The first vertical line is related to the global elastic state noted E in Fig. 9 and the second one to the global plastic state noted P in Fig. 9. Two different steps are observed in Fig. 13: first, a decrease of the temperature from the beginning of the test and then an increase of the temperature, beginning just before the first vertical line. This increase stops as the loading is interrupted.

\section{Discussion}

In this study, results are presented at two different states of deformation: when the sample is still globally elastic (instant $\mathrm{E}$ on

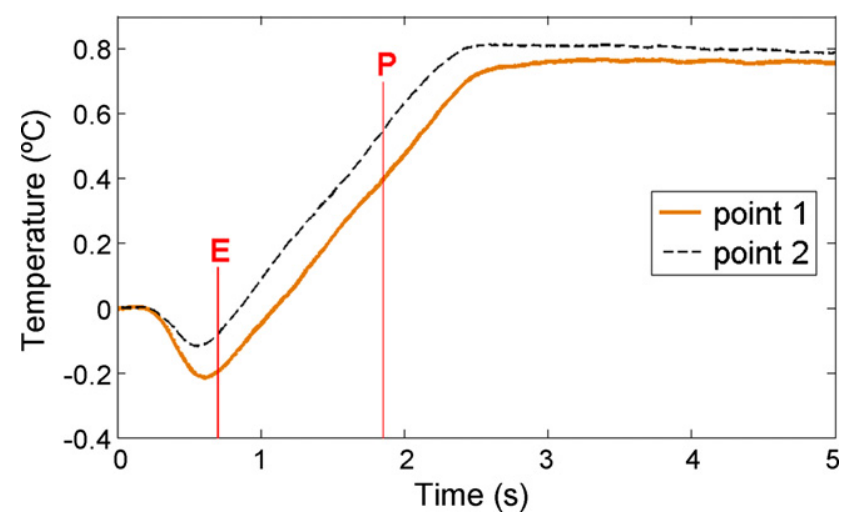

Fig. 13. Thermal evolutions of points 1 and 2 pinpointed in Fig. 10. The test is interrupted after $2.5 \mathrm{~s}$.

the stress-strain curve of Fig. 9) and once plasticity has occurred (instant P on the stress-strain curve of Fig. 9).

Both strain fields of Fig. 10 exhibit negative values whereas the sample is submitted to a uniform uniaxial tensile load. It can be seen on the histograms of Fig. 11 that these negative values only concern a small amount of ZOI among the studied zone, especially when the loading increases. As the grains have different crystallographic orientations, the effective stress to which they are really submitted depends on their orientation with respect to the loading axis and is also influenced by the neighbouring grains, especially if these grains are smaller than their neighbours. This could explain why some grains appear to be under compression. Nevertheless, to our knowledge, such a very local compressive state has never been reported and the knowledge of the microstructure of the studied zone would be here of great interest.

The strain field grabbed in the elastic domain (Fig. 10a) exhibits small levels of strains and looks quite homogeneous whereas in the plastic domain (Fig. 10b), levels of strains are much higher and clearly heterogeneous. This trend may appear more sharply thanks to the histograms of Fig. 11, which are plotted with the same strain scale. On the histogram of Fig. 11 a, the mean strain is $\varepsilon=0.10 \%$ with a narrow standard deviation equal to $0.29 \%$ whereas, on the histogram of Fig. 11b, the mean strain is $\varepsilon=1.71 \%$ with a much larger standard deviation equal to $1.07 \%$.

Points 1 and 2 account for the strain heterogeneities in the plastic domain since in Fig. 10b, the strain is $1.60 \%$ at point 1 and $4.64 \%$ at point 2 . This reveals that the strain state in plasticity is strongly different from one grain to another and that, according to their orientation with respect to the loading axis, some grains can be submitted to higher plastic strains than others. Here, once more, the knowledge of the local microstructure, as well as of the crystallographic orientations of the grains obtained for example by electron backscattered diffraction, could give interesting information. This will be the object of future works. Nonetheless both fields account for a localization of the plastic deformation at the scale of grains since the local strains are much higher than the global mean strain applied to the sample (Table 2 ).

Thermal fields representing $\Delta T(x, y, t)$ are plotted at instant $\mathrm{E}$ in the elastic domain (Fig. 12a) and at instant P in the plastic one (Fig. 12b). On both fields, it is possible to make out heterogeneities, but here, thermal heterogeneities between grains take place at the scale of two or three pixels. They are thus more awkward to be distinguished and to be related to the heterogeneities of the strain fields previously plotted in Fig. 10. These thermal fields show that the temperature over the gage zone (but it is also the case for the whole sample) decreases in the elastic domain. The mean temperature variation of Fig. $12 \mathrm{a}$ is actually $\Delta T=-0.11^{\circ} \mathrm{C}$. This is linked to 
Table 2

Results summary: strain and temperature variations at points 1 and 2, at instants $\mathrm{E}$ and $\mathrm{P}$.

\begin{tabular}{|c|c|c|c|c|c|}
\hline Instant studied & Global strain on the sample & Strain $\varepsilon$ at point 1 & Strain $\varepsilon$ at point 2 & Temperature variation $\Delta T$ at point 1 & Temperature variation $\Delta T$ at point 2 \\
\hline E (global elastic state) & $0.12 \%$ & $0.12 \%$ & $0.30 \%$ & $-0.19^{\circ} \mathrm{C}$ & $-0.07^{\circ} \mathrm{C}$ \\
\hline P (global plastic state) & $0.70 \%$ & $1.60 \%$ & $4.64 \%$ & $0.40^{\circ} \mathrm{C}$ & $0.56^{\circ} \mathrm{C}$ \\
\hline
\end{tabular}

the thermoelastic coupling effect which induces a reversible temperature raise of a sample submitted to an elastic compressive load and a temperature drop under elastic tensile load. In the plastic domain, the temperature of the sample increases, the mean temperature variation of Fig. 12b is indeed $\Delta T=0.51{ }^{\circ} \mathrm{C}$ over the gage zone. Here, the increase is due to the dissipation produced by the irreversible mechanisms of plasticity.

Since it is difficult to draw a parallel between the kinematic and thermal fields captured at instants $\mathrm{E}$ and $\mathrm{P}$, it is proposed to study the two points pinpointed on the strain fields of Fig. 10. As the strain field is given on the reference configuration, the positions of the initial material points are known, as well as their displacements thanks to DIC. This information allowed us to find the infrared detectors in front of which they respectively were all along the deformation of the specimen and then to plot in Fig. 13 the evolution of $\Delta T(x, y, t)$ of the material points 1 and 2 . The two curves plotted in Fig. 13 confirm the general thermal behaviour of the sample, which was already observed on the thermal fields of Fig. 12: the temperature evolves as soon as the sample is loaded; it is dropping during the elastic phase before rising as the sample undergoes plasticity. On these curves, it can be seen that, at instant $\mathrm{E}$, the temperature is increasing. This means that, at the end of the global elastic phase, dissipation and thus mechanisms of plasticity have already begun to occur locally, leading to a temperature raise. The temperature drop is different for points 1 and 2 . This may be explained by a local elastic anisotropy and by the different crystallographic orientations from one grain to another. At the moment marked $\mathrm{P}$ on the stress-strain curve (Fig. 9), point 2 exhibits a higher level of strain than point 1 (Fig. 10b) and its temperature variation is also higher than the one of point 1 (Fig. 13). On the one hand, Fig. 13 brings to the fore a thermal heterogeneity at the scale of grains, on the other hand it establishes a link between the plastic and thermal behaviours since the more plasticity a grain undergoes, the higher its temperature is. Table 2 summarizes the values of strain and temperature variations at points 1 and 2 at instants $E$ and $P$ and underscores the localization of strains as well as the link between the kinematic and thermal behaviours.

However, the thermal behaviours of other grains may not be always so clearly linked to the kinematic data. As a matter of fact, if a grain whose temperature is supposed to be low is surrounded by grains of higher temperatures, the diffusivity within the material makes the temperature of a cold point appear to be higher. Inversely, diffusion within the material also affects the temperature of the hotter points which then appear less hot than they are. The heat equation actually confirms that temperature is not an intrinsic data directly linked by the behaviour of the material but is highly affected by diffusion as well as by the environment. Hence some studies exist about the computing of thermal sources from the temperature fields, thanks to the heat equation, but this is not a trivial work since it consists in solving an inverse problem from discrete and noisy thermal data [23]. This will be the next step of this work.

\section{Conclusion}

For the first time, fully coupled full-field kinematic and thermal measurements have been performed on a steel at the scale of grains. All the technical difficulties to reach to a relevant experimental setup have been identified and overcome. This technique highlighted heterogeneities as well in the kinematic fields as in the thermal ones. In the tensile test presented here, the behaviour appears to be elastic at the structure scale whereas plasticity and thermal dissipation have already taken place within some grains. These first results are thus promising and can be of great interest to investigate metals behaviour in high cycle fatigue, where plasticity and damage are localized at the microstructure scale.

Nevertheless, even if fully coupled full-field measurements have been performed, it remains difficult to establish a direct link between both fields just by staring at them. Spatial heterogeneities are clearly visible over the strain fields especially once plasticity has taken place. It can be accounted for by the fact that the strain cumulates all along the deformation of the sample: the strain field at a given instant thus reflects all the strain history of the sample. On the contrary, the thermal information is instantaneous: it tends to diffuse within the sample as soon as it has appeared. As a consequence, observing the thermal fields at a given time is not systematically meaningful and more information can be extracted from thermal evolutions of different points, as it has been done in Section 3.3. Another way to investigate the irreversibilities and their link with strain fields is the inverse determination of thermal sources from the temperature fields, thanks to the heat equation; this would give access to intrinsic data of the material's behaviour.

However, whatever the way explored, it is not trivial to get the thermal evolution of a given material point since it moves during the tensile test. Nevertheless, it is considered to build an algorithm using the data given by digital image correlation in order to determine the displacement of every material point over the whole matrix of infrared detectors. This will then give access in the same time to the strain and temperature evolutions of the whole field of material points at the scale of grains. It will thus be possible to perform energy balances at the scale of grains, as it was formerly claimed by several authors [23].

\section{Acknowledgements}

This study has been funded by the CNRS, the Nord-Pas-de-Calais area (CISIT) and the European Community (FEDER). The authors would like to acknowledge the CNRS Research Group GDR's 2519 "Full-field measurements and Identification in Solid Mechanics" for its fruitful discussions as well as François Hild (LMT, Cachan) for its valuable advice about the software Correli ${ }^{\mathrm{LMT}}$. The "filter-mirror" has been developed by NOC Unlimited.

\section{References}

[1] J. Lemaitre, J.-L. Chaboche, Mechanics of Solid Materials, Dunod, Paris, 1988.

[2] B. Jaoul, Étude de la plasticité et application aux métaux, Dunod, Paris, 1965.

[3] W.S. Farren, G.I. Taylor, Proceedings of the Royal Society, London A 107 (1925) 422-451.

[4] G.I. Taylor, H. Quinney, Proceedings of the Royal Society, London A (1933) 307-326.

[5] A. Chrysochoos, Journal de Mécanique théorique et appliquée 4(1985) 589-614.

[6] W. Oliferuk, A. Korbel, W. Bochniak, Materials Science and Engineering A 319-321 (2001) 250-253.

[7] A. Chrysochoos, R. Peyroux, Revue Générale de Thermique 37 (1998) 582-606.

[8] P.R. Guduru, R.P. Singh, G. Ravichandran, A.J. Rosakis, Journal of the Mechanics and Physics of Solids 46 (1998) 1997-2016.

[9] P. Rosakis, A.J. Rosakis, G. Ravichandran, J. Hodowany, Journal of the Mechanics and Physics of Solids 48 (2000) 581-607.

[10] H. Ait-Amokhtar, C. Fressengeas, S. Boudrahem, Materials Science and Engineering A 488 (2008) 540-546. 
[11] B. Wattrisse, A. Chrysochoos, J.-M. Muracciole, M. Nemoz-Gaillard, European Journal of Mechanics A: Solids 20 (2001) 189-211.

[12] A. Chrysochoos, J.M. Muracciole, B. Wattrisse, Continuous Damage and Fracture (2000) 41-51.

[13] P.R. Guduru, A.J. Rosakis, G. Ravichandran, Mechanics of Materials 33 (2001) 371-402.

[14] H. Louche, A. Chrysochoos, Materials Science and Engineering A 307 (2001) $15-22$.

[15] A. Chrysochoos, Mecanique \& Industries 3 (2002) 3-14.

[16] V. Honorat, S. Moreau, J.M. Muracciole, B. Wattrisse, A. Chrysochoos, QIRT Journal 2 (2005) 153-172.

[17] H. Pron, C. Bissieux, QIRT Journal 1 (2004) 229-240.
[18] G. Gaussorgues, S. Chomet, Infrared Thermography, Chapman and Hall, London, 1994.

[19] F. Hild, B. Raka, M. Baudequin, S. Roux, F. Cantelaube, Applied Optics 41 (2002) 6815-6828.

[20] A. El Bartali, V. Aubin, S. Degallaix, Fatigue and Fracture of Engineering Materials and Structures 31 (2007) 137-151.

[21] J.F. Sacadura, Initiation Aux Transferts Thermiques, Lavoisier, Paris, 1982.

[22] OTUA, Propriétés physiques, in: Office Technique pour l'Utilisation de l'Acier, 2008.

[23] B. Berthel, A. Chrysochoos, B. Wattrisse, A. Galtier, Experimental Mechanics 48 (2008) 79-90. 